$10-9-2015$

\title{
Church Pew Exercise Integrated With Conventional Physical Therapy Following Total Knee Arthroplasty (TKA): Case Report
}

\author{
Randell Gonzales \\ University of Montana, randell_33@yahoo.com \\ Jerome Danoff \\ George Washington University, jdanoff@gwu.edu
}

Follow this and additional works at: https://nsuworks.nova.edu/ijahsp

Part of the Physical Therapy Commons, and the Physiotherapy Commons

\section{Recommended Citation}

Gonzales R, Danoff J. Church Pew Exercise Integrated With Conventional Physical Therapy Following Total Knee Arthroplasty (TKA): Case Report. The Internet Journal of Allied Health Sciences and Practice. 2015 Oct 09;13(4), Article 7.

This Manuscript is brought to you for free and open access by the College of Health Care Sciences at NSUWorks. It has been accepted for inclusion in Internet Journal of Allied Health Sciences and Practice by an authorized editor of NSUWorks. For more information, please contact nsuworks@nova.edu. 


\title{
Church Pew Exercise Integrated With Conventional Physical Therapy Following Total Knee Arthroplasty (TKA): Case Report
}

\begin{abstract}
Background and Purpose: Physical therapy treatment following Total knee Arthroplasty (TKA) consists of a combination of strengthening and range of motion exercises. The exercise technique, church pew exercise (CPE), has been proposed to enhance quadriceps facilitation and improve function. This is a technique that has the patient standing and rocking forward/back. The backward motion is arrested by engagement of the upper calf against a solid object, creating a sudden flexion torque at the knee and a sudden extension torque at the hip. The combination of CPE with standard physical therapy is claimed to provide better quadriceps control and faster walking post TKA. In this case study, a 51-year-old female with decreased knee active and passive range of motion (ROM), decreased lower extremity (LE) muscle strength, and limited ambulation post left knee TKA, received conventional physical therapy treatment plus CPE. Methods: The patient received 11 physical therapy home sessions. The first 8 sessions were used to strengthen her knee and increase active and passive ROM. The CPE intervention was performed 3 weeks post-surgery, during the last 3 physical therapy sessions. At these three sessions the patient performed the Timed Up and Go (TUG) before and after CPE. Outcomes: At initial evaluation, 4 days post left knee TKA, this patient's left knee active ROM was only 15 to 76 degrees, and manual muscle testing at her hip and knee indicated strengths of 2- (hip flexors), 3- (hamstrings and quadriceps), and 3+ (hip abductors), all out of 5 , and her times on two trials of the TUG were 30.31 and $30.65 \mathrm{sec}$, indicating impaired functional ability. At all CPE sessions (3 weeks post-surgery) the patient demonstrated increased gait speeds (i.e. shorter times) on the TUG after the CPE (pre CPE mean $=13.2 \mathrm{sec}$; post CPE mean = 11.2), increased stance time on her affected lower extremity, and increased step length on her contralateral lower extremity. The patient reported increased knee stability. Clinical Relevance: Rationale for CPE is based on neurological facilitation of quadriceps and other lower extremity muscles. However, to engage in this exercise the patient must have the ability to balance and to control the hip and knee joints. This patient required several weeks of strengthening and active and passive ROM exercises before she could perform the CPE. Once able to perform the CPE, she demonstrated improvements in her walking ability, and she expressed greater confidence. Conclusion: Provided a minimal level of strength and active and passive ROM has been attained, CPE may be a valuable short-term supplement to current strengthening regimens addressing quadriceps functional deficits following TKA. Other conditions that involve gait problems related to quadriceps insufficiency might also benefit. The long-term efficacy of CPE remains to be determined.
\end{abstract}




\title{
TIAHSP \\ The Internet Joưnal of Allied Health Sciences and Practice \\ Dedicated to allied health professional practice and education \\ Vol. 13 No. 4 ISSN 1540-580X \\ Church Pew Exercise Integrated With Conventional Physical Therapy Following Total Knee Arthroplasty (TKA): Case Report
}

\author{
Randell Gonzales, DPT 1 \\ Jerome V. Danoff, PT, PhD2
}

1. Senior Staff Therapist, Central Georgia Home Health, The Medical Center of Georgia, Macon, Georgia

2. Professor and Vice Chairman, Department of Exercise and Nutrition Sciences, George Washington University, Milken Institute School of Public Health, Washington, DC

United States

\begin{abstract}
Background and Purpose: Physical therapy treatment following Total knee Arthroplasty (TKA) consists of a combination of strengthening and range of motion exercises. The exercise technique, church pew exercise (CPE), has been proposed to enhance quadriceps facilitation and improve function. This is a technique that has the patient standing and rocking forward/back. The backward motion is arrested by engagement of the upper calf against a solid object, creating a sudden flexion torque at the knee and a sudden extension torque at the hip. The combination of CPE with standard physical therapy is claimed to provide better quadriceps control and faster walking post TKA. In this case study, a 51-year-old female with decreased knee active and passive range of motion (ROM), decreased lower extremity (LE) muscle strength, and limited ambulation post left knee TKA, received conventional physical therapy treatment plus CPE. Methods: The patient received 11 physical therapy home sessions. The first 8 sessions were used to strengthen her knee and increase active and passive ROM. The CPE intervention was performed 3 weeks post-surgery, during the last 3 physical therapy sessions. At these three sessions the patient performed the Timed Up and Go (TUG) before and after CPE. Outcomes: At initial evaluation, 4 days post left knee TKA, this patient's left knee active ROM was only 15 to 76 degrees, and manual muscle testing at her hip and knee indicated strengths of 2- (hip flexors), 3- (hamstrings and quadriceps), and 3+ (hip abductors), all out of 5, and her times on two trials of the TUG were 30.31 and $30.65 \mathrm{sec}$, indicating impaired functional ability. At all CPE sessions (3 weeks post-surgery) the patient demonstrated increased gait speeds (i.e. shorter times) on the TUG after the CPE (pre CPE mean $=13.2 \mathrm{sec}$; post CPE mean $=11.2$ ), increased stance time on her affected lower extremity, and increased step length on her contra-lateral lower extremity. The patient reported increased knee stability. Clinical Relevance: Rationale for CPE is based on neurological facilitation of quadriceps and other lower extremity muscles. However, to engage in this exercise the patient must have the ability to balance and to control the hip and knee joints. This patient required several weeks of strengthening and active and passive ROM exercises before she could perform the CPE. Once able to perform the CPE, she demonstrated improvements in her walking ability, and she expressed greater confidence. Conclusion: Provided a minimal level of strength and active and passive ROM has been attained, CPE may be a valuable short-term supplement to current strengthening regimens addressing quadriceps functional deficits following TKA. Other conditions that involve gait problems related to quadriceps insufficiency might also benefit. The long-term efficacy of $\mathrm{CPE}$ remains to be determined.
\end{abstract}

\section{BACKGROUND}

Total knee arthroplasty (TKA) or total knee replacement is a surgical technique to replace damaged weight bearing surfaces of the knee joint with metal and plastic components in order to relieve pain and disability, correct deformity, and restore normal activities of daily living. ${ }^{1}$ The need for TKA may be due to arthritic conditions including osteoarthritis, rheumatoid arthritis, and post-traumatic arthritis. ${ }^{2}$ 
Non-surgical treatments include weight loss, aerobic exercise, osteopathic manipulative treatment, non-steroidal antiinflammatory drugs, and corticosteroid injections. ${ }^{3}$ Also often used are bracing, ambulatory aides, and taping. ${ }^{2-4}$ If these nonsurgical interventions are insufficient to relieve pain, and the patient's ability to perform tasks such as walking, getting in or out of a chair, and climbing stairs has deteriorated, TKA will be considered. ${ }^{1}$

In an individual for whom TKA is recommended, the strength of the quadriceps muscle is almost always found to be low. ${ }^{6-13}$ This weakness may be due to an extended period of time with knee pain and the associated reduction of voluntary muscle activation. This combination may result in muscle inhibition and quadriceps atrophy. In addition, hamstrings strength has been known to decrease by as much as 50\% one month after TKA. ${ }^{3}$ Voluntary quadriceps activation and hamstrings strength are important in rehabilitation after TKA since effectiveness of exercises and thus, the progression of physical function, is related to lower extremity strength. $6,8,10$

Current physical therapy treatments post-TKA are intended to improve lower extremity function. These may include pain modalities, range of motion exercises, patella-femoral joint mobilization, general lower extremity strengthening, functional training, and specific quadriceps strengthening exercises. Quadriceps strengthening usually includes short and long arc knee extensions, quadriceps setting, close chain knee extensions, isokinetic knee extensions, and neuromuscular electrical stimulation (NMES). 1,2,4-6

TKA patients who have received conventional therapy often still demonstrate marked decreases in gait speeds and reduced ability to climb stairs one year after surgery compared to age-matched, healthy individuals. 2,5 However, there is some evidence that high intensity volitional strength training and NMES, either separately or in combination will reverse deficits in quadriceps muscle mass, improve quadriceps activation, and add to the benefits of strengthening exercises.1,2,6,14 These results suggest a possible improvement in the management of quadriceps weakness and atrophy may be achieved by using progressive quadriceps strengthening regimens to facilitate functional recovery.

The church pew exercise (CPE) is a therapeutic exercise construct designed to specifically facilitate quadriceps contraction. ${ }^{15}$ O'Halloran claims in unpublished research that TKA patients who performed these exercises exhibited increases in walking speeds and decreased stair climbing times as determined by the Ten Meter Walk Test (TMWT) and the 12 Step Stair Test, respectively, compared to the measurements before performing the CPE. ${ }^{15}$ O'Halloran suggests that these improvements in walking speed and stair climbing ability are due to facilitation of involuntary quadriceps contraction resulting in neuromuscular drive to the lower extremity kinetic chain, therefore enhancing the effectiveness of common strengthening exercises in TKA protocols. Also, O'Halloran believes that CPE enhances ankle mobility, improving necessary and natural movements in the kinetic chain such as stride length, cadence and proprioception. The CPE has not been tested in large scale clinical research, but O'Halloran's initial unpublished findings are consistent with the studies of Petterson et al and Bade and StevensLapsley.4,15,16

Church pew exercises consist of having the patient in erect stance, closely positioned in front of a standard folding chair (or any device having an edge at the height of the patient's upper calf, hence the name "church pew"). The patient then rocks forward and backward. The forward motion is arrested by a rapid contraction of the plantar flexor muscles; the trunk extensors also become active at this time. The backward motion is arrested by the calf making contact with the chair. This creates a sudden flexion torque at the knee and an extension torque at the hip, facilitating a rapid response in the quadriceps and hip flexors, respectively. Visualization of these actions can be seen in Figures.1-5 (next page).

Although NMES has been used successfully to facilitate quadriceps function and improve gait post TKA, CPE would have an advantage over NMES in terms of cost effectiveness because a patient or the therapist would not need to purchase or rent an NMES unit.8,17-19 In addition, a patient, after proper training, could safely engage in CPE independently. Therefore, if CPE were beneficial for functional recovery after TKA, it might obviate the need for NMES.

(C) The Internet Journal of Allied Health Sciences and Practice, 2015 


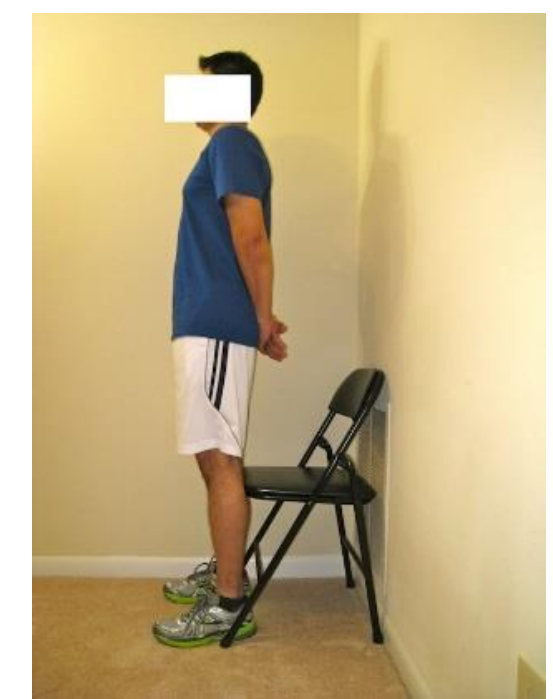

Figure 1.

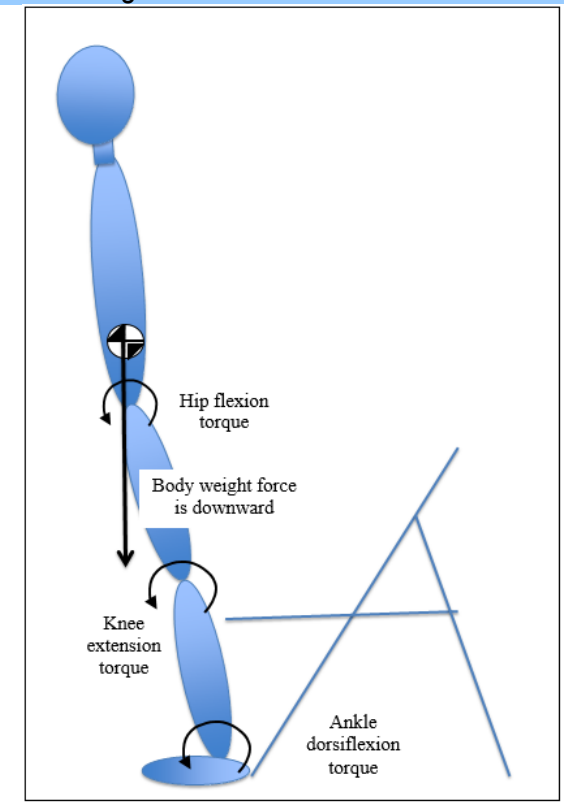

Figure 4.

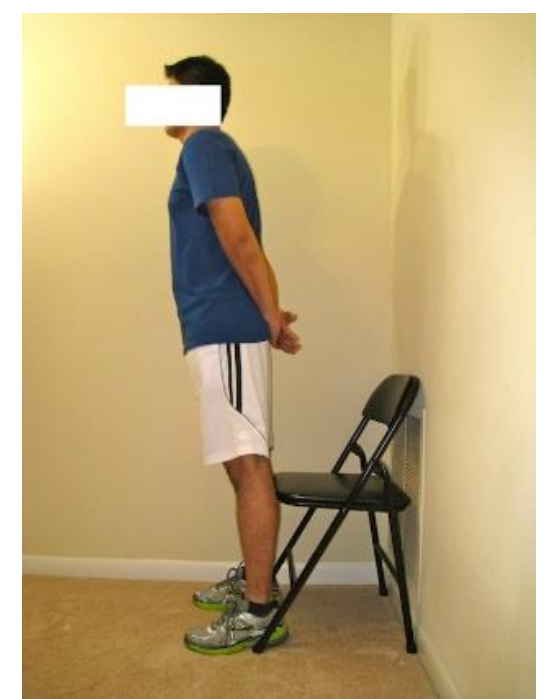

Figure 2.

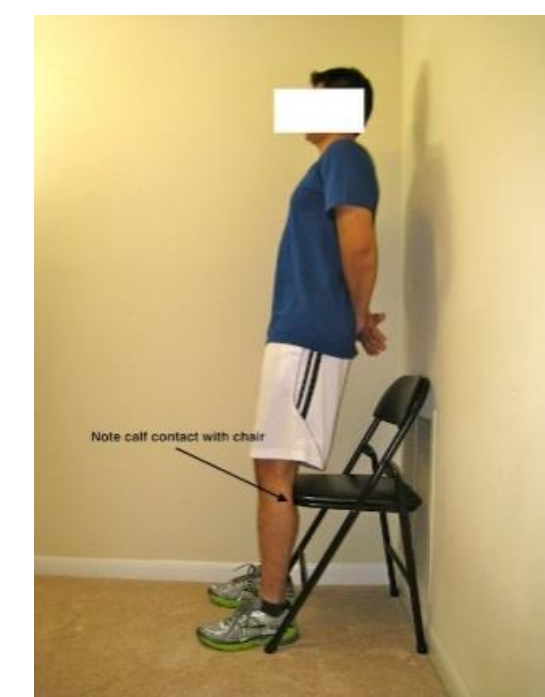

Figure 3.

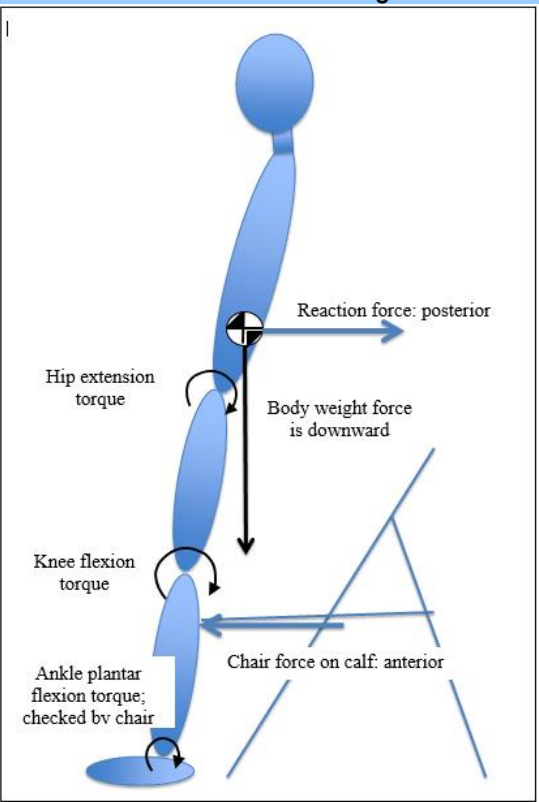

Figure 5.

This case report will discuss the evaluation and management of a patient with TKA combining the CPE with traditional quadriceps exercises. The purposes of this case report are to describe the effects of CPE on physical performance and quality of gait when used as an adjunct therapy to conventional strengthening and stretching physical therapy.

\section{CASE DESCRIPTION}

The patient was a 51-year-old female, non-smoker and non-drinker, with a history of hypertension, type 2 diabetes mellitus and hyperlipidemia. She had been taking a pill to manage her diabetes. She had degenerative joint disease and a previous right TKA, followed 3 months later by an unscheduled left TKA (Biomet Orthopedics, Inc. Warsaw, IN) due to worsening degenerative joint disease that made her left knee stiff and painful, and ambulation impossible. Despite drug management (Lortab@ acetaminophen plus hydrocodone), her left knee pain had become intolerable.

The patient underwent a physical therapy home evaluation 4 days post the left knee TKA. At that time, she reported stiffness of her left knee joint and throbbing pain along the anterior aspect of the left knee at the surgical incision site. A Visual Analog Scale (VAS) was used to assess pain level.20,21 With 0 (no pain) and 10 (worst possible pain), she indicated her pain was 2 at rest but increased to 4 with motion or weight bearing activities. She admitted that her pain level had been 8 before taking her pain 
medication. The patient also reported limitation in ability to perform household chores, bathing, standing, prolonged ambulation, and stepping up at her kitchen and front door entrances.

During the physical therapy evaluation, this patient was limited by pain and joint stiffness, required use of an assistive device, and had balance deficits and gait deviations. Physical therapy goals included return to work, ability to do housework, and ability to assist her significant other.

\section{Examination}

Range of Motion (ROM). Patient's left knee extension/flexion active ROM was measured in supine position with a large arm goniometer. (Use of the large arm goniometer to measure knee ROM has been considered reliable since the 1980's with $r$ and ICC .09.22) Pain was elicited in left knee with overpressure in both extension and flexion. Significant limitation was apparent as patient's active knee ROM was only 15 to 76 degrees during evaluation. In comparison active ROM for an unaffected knee is around $0-130$ degrees.

Muscle Strength. Lower extremity muscle performance was assessed by utilizing manual muscle testing (MMT). ${ }^{23}$ The results were the following: left lower extremity: hip flexors $2-15$, hip abductors $3+/ 5$, hamstrings and quadriceps $3-/ 5$; right lower extremity: hip flexors $4+/ 4$, hip abductors $4 / 5$, hamstrings and quadriceps $4+/ 5$. Both upper extremities were grossly graded $5 / 5$.

Functional Mobility Assessment. Timed Up and Go Test (TUG): patient completed 2 trials with use of a rolling walker. On the 1st trial, patient took 30.31 seconds to complete the test, and 2nd trial, 30.65 seconds. These times indicate impaired functional mobility. Times of 11 to 20 seconds are considered within normal limits for frail elderly or for persons with disability; scores of more than 20 seconds indicate functional mobility impairment. ${ }^{24}$ Interrater and test-retest reliability of TUG are very high (0.98) ${ }^{25}$

Gait Assessment. The patient used a rolling walker to ambulate. She demonstrated decreased stance time on left lower extremity, suggesting painful knee with weight bearing. Also observed were inadequate left knee flexion during swing phase, decreased heel strike, short step length, and visible terminal knee extension deficit at end of stance phase. Base of support was wide and cadence was low.

Functional Measures. The Lower Extremity Functional Scale (LEFS), a self-reporting instrument, was utilized to establish a baseline functional level. Binkley et al concluded that LEFS is reliable and can be used for clinical decision making with testretest reliability of $0.94 .{ }^{26} \mathrm{~A}$ score of $80 / 80$ indicates best function, and the lower the score, the lower the function. This patient completed the 20-item questionnaire with a score of only 7/80. A Stair Climb Test would also have been useful for evaluating patient performance, but this patient was receiving home-care physical therapy, and there were no flights of stairs in this patient's home.

Clinical Impression. Prior to the left TKA this patient was independent in activities of daily living (ADLs) and was able to ambulate for a few blocks. At the time of physical therapy examination, patient demonstrated limitations in her ability to perform transfers and to ambulate, and she reported difficulty in most ADLs. This decline was due to pain, stiffness/decreased range of motion, and decreased muscle strength around her left knee, altered gait mechanics, and decreased balance. The patient's diagnostic classification was Musculoskeletal Pattern 4H: Impaired Joint Mobility, Motor Function, Muscle Performance, and Range of Motion Associated with Joint Arthroplasty (Guide to Physical Therapy Practice). ${ }^{27}$

The patient expressed a strong desire to get well, be independent in ADLs, and return to her previous level of function. Given her clinical presentation, situation, and goals, physical therapy intervention was deemed necessary. Due to the gait deviations observed, functional limitation, significant limitation on left knee ROM, and other pertinent measures, the patient was considered an appropriate candidate for the use of CPE as an intervention for neuromuscular quadriceps facilitation.

\section{Intervention}

The patient received physical therapy treatment at home 2 to 3 times per week for a total of 11 visits. During the first 5 visits (weeks 1 and 2), physical therapy intervention emphasized ROM, both active and passive, to maximize knee flexion and extension. Exercises utilized are listed in Table 1. The patient was not able to tolerate closed chain left knee extension at this time. Ice was utilized after exercise with leg elevation for 20 minutes to manage pain and swelling.

(C) The Internet Journal of Allied Health Sciences and Practice, 2015 
TABLE 1. Exercises Used for Physical Therapy Home Care of TKA Patient

\begin{tabular}{|l|l|}
\hline \multicolumn{1}{|c|}{ Exercise } & \multicolumn{1}{c|}{ Purpose } \\
\hline heel slides & quadriceps/hamstring reciprocation \\
\hline seated and standing leg curls & hamstring strengthening \\
\hline patellar mobilization & Range of Motion (ROM) \\
\hline low-load, long-duration stretching & Range of Motion (ROM) \\
\hline quadriceps sets & quadriceps strengthening \\
\hline short arc extensions & quadriceps strengthening \\
\hline straight leg raises & quadriceps strengthening \\
\hline repeated sit-to-stands & quadriceps strengthening \\
\hline hip abduction, adduction & hip control \\
\hline ankle plantarflexion and dorsiflexion & ankle control \\
\hline
\end{tabular}

During visits 6 to 8 , elastic bands were added to provide resistance for the quadriceps. Closed kinetic chain terminal knee extension exercises were also added.

\section{Administration of Church Pew Exercises}

CPE did not start until the 9th visit, 3 weeks following left TKA. According to O'Halloran, most TKA patients, at less than 3 weeks post-surgery, are unable to stand evenly and achieve terminal knee extension. ${ }^{15}$ This was the case with this patient. Terminal knee extension is necessary to fully sway back and forth when executing the CPE correctly. To prepare the patient for CPE, emphasis at the end of week 2 was placed on passive long-duration stretch towards terminal left knee extension. ${ }^{28}$ The patient received both a visual demonstration and verbal instruction. In conjunction with the CPE (visits 9, 10,11) a TUG test (two trials) using a single point cane was executed before and after each CPE. ${ }^{25}$ TUG results are shown in Table 2.

Table 2. Patient's Walking Performance after Incorporation of Church Pew Exercise (CPE) Into Physical Therapy Treatment

\begin{tabular}{|l|l|l|l|}
\hline PT Home Visit & \multicolumn{2}{|l|}{$\begin{array}{l}\text { Timed Up and Go Test, } \\
\text { Mean of 2 trials (seconds) }\end{array}$} & Patient Comment \\
\hline & Before CPE & After CPE & \\
\hline 9 & 14.6 & 11.6 & "feels stiff but stable on left knee" \\
\hline 10 & 10.8 & 9.9 & "feels more stable on left knee" \\
\hline 11 & 14.1 & 12.0 & $\begin{array}{l}\text { "feel more confident putting weight on } \\
\text { left leg" }\end{array}$ \\
\hline
\end{tabular}

Note: Low-load, long-duration stretch towards left knee extension was applied before CPE during Visits 9 and 10 and after CPE during Visit 11.

To begin CPE, a standard chair was positioned securely against a wall. The patient stood in front of the chair with both of her upper calf muscles against the chair and began a controlled sway, first shifting her weight forward onto her big toes and then swaying back, shifting her weight onto her heels, until her calves again contacted the chair edge (Figs. 1-3; note: model in photos is not the patient). The duration of this exercise is usually around 3 minutes. For this patient, the duration was extended to 4 minutes, as the first minute of exercise was used to correct the patient's position and movement (to eliminate bending from the hips). A more advanced variant of the CPE may be achieved by placing only the affected calf against the chair (instead of both calves), and by raising both upper extremities and performing diagonal reaches back and forth to change center of mass. ${ }^{9}$ this patient did not perform the advanced variant of the CPE.

Full description of the CPE can be found at the web link "The Church Pew Exercise: Joint Replacement Quadricep Facilitation": $<$ http://www. youtube.com/watch?v=umpwihnctic $>$ Our interpretation of the CPE is as follows: during the forward sway motion of the CPE, there is a flexion torque at the hip joint and an extension torque at the knee joint. This combination of torques will stimulate a strong response in the hamstring muscles which are rapidly stretched at this time to control hip flexion and resist knee hyperextension. Concurrently, there is a large dorsiflexion torque that will elicit a strong and rapid response from the triceps surae (Fig.4). During the backward sway, movement is abruptly stopped as the upper calf and leg contact the chair edge (similar to leaning back against a church pew). This will result is a large and sudden knee flexion torque and a large hip extension torque requiring strong and rapid responses from the quadriceps and hip flexors, respectively (Fig.5). These reactions are further reinforced by neurological input associated with balance and postural adjustments. The abruptness or sudden development of these torques and concomitant responses is more important than the magnitudes of the forces developed because simple force 
development (or strength) can be achieved through many exercises, but quick application of force depends on a rapid neurological response. Throughout the sway motions, the hip and knee joints remain close to full extension, similar to the pattern of the lower extremity during the mid-stance phase of gait, which is a critical weight-bearing phase of the gait cycle. Therefore, the CPE elicit desirable neurological patterns in postural positions closely aligned with those during gait.

Immediately after the CPE, two additional trials of TUG were performed to test the claim that walking would improve after CPE. She completed the test with markedly decreased time compared to before the CPE (Table 2). The patient reported that her left knee felt stiff but stable after completing the CPE. After the TUG trials, left knee flexion ROM was further addressed for the remaining treatment time using contract-relax ${ }^{24}$ and step stretching. The patient's left knee active ROM after treatment was 7 to 87 degrees.

On the 10th visit, the patient denied any pain/discomfort on her left knee, but active ROM before treatment was still limited to 10 to 85 degrees. The patient tolerated 4 minutes of low-load, long-duration stretch towards knee extension. Similarly to visit 9 , this was followed by two trials of TUG, three minutes of CPE, and two more trials of TUG while using a single point cane. During the first two TUG trials (pre CPE), there was a slight knee buckle and visible terminal knee extension lag. During the last two TUG trials (post CPE), the patient demonstrated increased stance time on her left lower extremity, and her right step length increased. She stated that she felt increased stability of her left knee during stance phase. Once again, gait velocity was markedly increased after CPE as evidenced by reduced TUG times (Table 2). Contract-relax stretching was repeated to increase knee flexion ROM, and active ROM after treatment was 6 to 89 degrees.

On the last physical therapy session, TUG and CPE were executed similarly to visits 9 and 10 . Again the patient completed the test faster after CPE compared to measurements before CPE. The patient noted increased stability and reported greater confidence with weight bearing on her left lower extremity after the intervention. Increased step length on the right lower extremity was observed. The patient was cleared to start outpatient therapy the following week.

\section{Outcomes}

Table 2 is a summary of TUG results before and after CPE. All three trials demonstrated an increase in walking speed after CPE performance based on completing the TUG in less time compared to time before CPE. Patient consistently reported her knee feeling "stable" after completing CPE. During gait assessment the patient demonstrated an increase in stance time on left lower extremity, increase step length on right lower extremity, and increased walking speed.

LEFS scores, ROM, MMT scores, and VAS are listed in Table 3. The patient's LEFS self-report function increased by 32 scale points. A difference of more than 9 points represents a true change, so this patient clearly improved her general lower extremity function. ${ }^{26}$ She reported improvement in performance of housework, ambulation in the house, putting on shoes or socks, and getting in and out of the car. However, no changes in more demanding activities such as running or hopping were reported. Left knee active ROM and quadriceps and hamstrings strength all demonstrated improvement at discharge (transition to outpatient therapy). Pain level was only slightly higher at discharge with a report of morning stiffness, but this might have been due to the increased activity by this patient.

Table 3. Outcome Measures, Pre and Post Physical Therapy Intervention

\begin{tabular}{|l|l|l|}
\hline & Initial Evaluation & Discharge \\
\hline Lower Extremity Functional Scale (LEFS) & $7 / 80$ & $39 / 80$ \\
\hline Active Range of Motion (ROM) & 15 to 76 degrees & 7 to 87 degrees \\
\hline Manual Muscle Testing (MMT) & $\begin{array}{l}\text { Left quadriceps 3-/5 } \\
\text { Left hamstrings 3-/5 }\end{array}$ & $\begin{array}{l}\text { Left quadriceps 4/5 } \\
\text { Left hamstrings 4/5 }\end{array}$ \\
\hline Pain (VAS) & $8 / 10$ & $5 / 10$ \\
\hline
\end{tabular}

\section{DISCUSSION}

In this case report CPE was used as a supplement to standard physical therapy for a patient, post TKA, receiving home-care physical therapy. Standard treatments for TKA rehabilitation focus on increasing knee ROM, increasing quadriceps and hamstrings strength, and providing patient education for safe mobility practices. 2,4,7,16,24,29 $\mathrm{CPE}$ as an adjunct treatment is believed to facilitate quadriceps function and should manifest itself via faster walking.

As a consequence of the swaying motion of the $\mathrm{CPE}$, the quadriceps, hamstring, and triceps surae muscles are stimulated to rhythmically vary their contraction pattern in response to the shifting balance of the body and the torques at the hip, knee, and ankle joints. Facilitation of the hip flexors and extensors and plantarflexors will contribute to improved muscle control during gait. However, of particular importance is the activation of the quadriceps, which occurs very rapidly due to the sudden arrest of the

(c) The Internet Journal of Allied Health Sciences and Practice, 2015 
backward movement by striking the chair edge just below the knee. This facilitates neuromuscular activation of the quadriceps and prepares this muscle to function appropriately during heel strike when quick response is required to avoid collapse or falling.

In addition, the postural patterns used with the CPE are very similar to the lower extremity patterns during the mid-stance phase of the gait cycle. During ambulation, the transition from heel strike to mid-stance is the period when an individual with compromised quadriceps function is most at risk of losing control and falling. Therefore, facilitating these activity patterns immediately before an individual is about to walk may augment lower extremity function and provide a greater sense of confidence in that person.

Stair climbing presents greater difficulty than gait on a level surface, and testing of stair climbing ability would be valuable for assessing functional ability. However, in this patient's home setting, stair climbing was unavailable, so gait observation and TUG were used to determine functional abilities. She did have two steps at the entrance of her home, and she claimed to have no difficulty in managing these steps by the time she was discharged to out-patient physical therapy. We believe that the LEFS also provides an indication of the patient's functional independence. Although LEFS is a self-report, and, therefore, not fully objective, this instrument suggested that our patient had improved at the time of discharge compared to initial evaluation. Finally, VAS indicated a decrease in pain, and while this is not a functional measure, it did correspond to the patient's increasing confidence with her ability to walk independently.

For the first 8 home visits ( $<3$ weeks post-surgery), stretching and strengthening were utilized to work towards terminal knee extension. Until a patient can achieve close to full active knee extension and fully tolerate this type of activity, CPE is not possible. This is because of the lower extremity positioning needed for CPE. This patient was able to acceptably perform CPE during her final three home visits $(\# 9,10,11)$, and her responses to the CPE were positive. We do not believe that CPE would have been appropriate for this patient at an earlier time in her therapy.

The most direct indicators of whether CPE influenced this patient's gait ability were the times for the TUG. At each of these final three visits, the patient took two trials of the TUG, engaged in three minutes of CPE, then two more trials of TUG. In all three comparisons, and for all trials, the results were clear that the patient walked faster after the CPE. She also commented that she felt better (i.e. more in control) after the CPE. We believe that this increased confidence was because her knee extensors were now better prepared to quickly accept the forces of heel strike and weight bearing.

This patient's outcome was similar to the subjects in O'Halloran's initial unpublished investigation of CPE who demonstrated acute increases in walking and stair climbing speeds (TMWT and Stair climbing test, respectively). ${ }^{15}$ Based on the LEFS, this patient's lower extremity function clearly improved.

We believe that this patient demonstrated reasonable improvement in functional abilities based on a course of standard physical therapy home treatment after her TKA. However, the addition of CPE when she was able to achieve the necessary terminal knee extension appeared to further enhance her walking ability. She was walking more comfortably and faster after each CPE administration. Any patient with instabilities of the LE, particularly those related to loss of control of the knee from heel strike to mid-stance should benefit from CPE. These exercises have a built-in safety factor when performed as described because when the patient sways backward, if the knee gives out, the patient would simply sit back into the chair.

\section{CONCLUSION}

These results suggest that CPE in addition to traditional therapy can improve gait speed, at least for the short term. Thus, it may be a useful supplement to current physical therapy regimens for patients following TKA. Before using CPE, the patient must achieve adequate knee extension, be able to stand symmetrically, and be able to correctly execute the exercise. Since the principal contribution of CPE is to facilitate rapid quadriceps activation, this might be useful for other conditions in which the patient has not been ambulating successfully - with the caveat that the patient has adequate knee extension and the ability to stand erect. CPE does not require any special equipment, is safe to perform, and can easily be taught to patients. Further data will be needed to determine if the positive outcomes of CPE will persist long term, if longer or more frequent bouts of CPE would be advantageous, and if CPE has an influence on long-term recovery after TKA.

\section{REFERENCES}

1. American Academy of Orthopeaedic Surgeons. Total Knee Replacement. December 2011. http://orthoinfo.aaos.org/topic.cfm?topic=a00389. Accessed February 1, 2013.

2. Van Manen M, Nace J, Mont MA. Management of primary knee osteoarthritis and indications for total knee arthroplasty for general practitioners. J Amer Osteo Assn. 2012;112(11):709-15. [PMID: 23139341]

(c) The Internet Journal of Allied Health Sciences and Practice, 2015 
3. McKinnis L. Fundamentals of Musculoskeletal Imaging. Ch: Degenerative Joint Disease, 4e. Philadelphia, PA. FA Davis Company, 2014.

4. Petterson SC, Mizner RL, Stevens JE, Raisis L, Bodenstab A, Newcomb W, Snyder-Mackler L. Improved function fROM progressive strengthening interventions after total knee arthroplasty: A randomized clinical trial with an imbedded prospective cohort. Arthritis Rheum. 2009;61(2):174-83. [PMID: 19177542]

5. Walsh M, Woodhouse L, Thomas S, Finch E. Physical impairments and functional limitations: A comparison of individuals 1 year after total knee arthroplasty with control subjects. Phys Ther. 1998;78:248-58. [PMID: 9520970]

6. Mizner R, Petterson S, Snyder-Mackler L, Stevens J, Vandenborne K. Early Quadriceps Strength Loss After Total Knee Arthroplasty: The Contributions of Muscle Atrophy and Failure of Voluntary Muscle Activation. J Bone Joint Surg Am. 2005;87(5):1047-53. [PMID: 15866968]

7. Bell KA, Shovestul A. Total Knee Arthroplasty. Clinical Summary. PT Now. October 2011. (based on Bade M, Bell KA, Stevens J, Manal TJ. Joint Arthroplasty: Advances in Surgical Management and Rehabilitation. Independent Study Course 20.2.2. Total Knee Arthroplasty. APTA Orthopedic Section Monograph, 2010.)

8. Stevens JE, Mizner RL, Snyder-Mackler L. Neuromuscular electrical stimulation for quadriceps muscle strengthening after bilateral total knee arthroplasty: a case report. J Orthop Sports Phys Ther. 2004;34:21-9. [PMID: 14964588]

9. Silva M, Shepherd EF, Jackson WO, Pratt JA, McClung CD, Schmalzried TP. Knee strength after total knee arthroplasty. J Arthroplasty. 2003;18(5):605-11. [PMID: 12934213]

10. Stevens JE, Mizner RL, Snyder-Mackler L. Quadriceps strength and volitional activation before and after total knee arthroplasty for osteoarthritis. J Orthop Res. 2003;21(5):775-9. [PMID: 12919862]

11. Jaggers JR, Simpson CD, Frost KL, Quesada PM, Topp RV, Swank AM, Nyland, JA. Prehabilitation before knee arthroplasty increases postsurgical function: a case study. J Strength and Conditioning Res. 2007;21(2):632-4. [PMID: 17530958]

12. Mizner RL, Petterson SC, Stevens JE, Axe MJ, Snyder-Mackler L. Preoperative quadriceps strength predicts functional ability one year after total knee arthroplasty. J Rheum. 2005;32(8):1533-9. [PMID: 16078331]

13. van Leeuwen DM, de Ruiter CJ, Nolte PA, de Haan A. Preoperative Strength Training for Elderly Patients Awaiting Total Knee Arthroplasty. Rehab Res Prac. 2014;2014:462750. Epub 2014 Feb 13. (http://dx.doi.org/10.1155/2014/462750) [PMID: 24693435]

14. Stevens-Lapsley J, Balter J, Kohrt W, Eckhoff D. Quadriceps and Hamstrings Muscle Dysfunction after Total Knees Arthroplasty. Clinical Orthopaedics and Related Research. 2010;468:2460-8. [PMID: 20087703]

15. O'Halloran J. The Initial Investigation of The Church Pew Exercise (CPE) to Facilitate Quadriceps Function Following Total Knee Arthroplasty. (Unpublished research) http://www.cyberpt.com/ohalloranquadricepchurchpew.asp. Accessed February 1, 2013.

16. Bade M, Stevens-Lapsley J. Early High Intensity Rehabilitation Following Total Knee Arthroplasty Improves Outcomes. J Orthop Sports Phys Ther. 2011;41(12):932-41. [PMID: 21979411]

17. Mintken PE, Carpenter KJ, Eckhoff D, Kohrt WM, Stevens JE. Early neuromuscular electrical stimulation to optimize quadriceps muscle function following total knee arthroplasty: a case report. J Orthop Sports Phys Ther. 2007;37:364-71. [PMID: 17710905]

18. Petterson S, Snyder-Mackler L. The use of neuromuscular electrical stimulation to improve activation deficits in a patient with chronic quadriceps strength impairments following Total Knee Arthroplasty. J Orthop Sports Phys Ther. 2006;36(9):678-85. [PMID: 17017273]

19. Stevens-Lapsley J, Balter J, Wolfe P, Eckhoff D, Kohrt W. Early Neuromuscular Electrical Stimulation to Improve Quadriceps Muscle Strength After Total Knee Arthroplasty: A Randomized Controlled Trial. Phys Ther. 2012;92(2):21026. [PMID: 22095207]

20. Banos JE, Bosch F, Canellas M, Bassols A, Ortega F, Bigorra J. Acceptability of visual analog scales in the clinical setting: a comparison with verbal rating scales in postoperative pain. Methods Find Exp Clin Pharmacol. 1989;11(2):123-7. [PMID: 2709918]

21. Roach KE, Brown MD, Dunigan KM, Kusek CL, Walas M. Test- retest reliability of patient reports of low back pain. $J$ Orthop Sports Phys Ther. 1997;26(5):253-9. [PMID: 9353688]

22. Norkin C, White J. Measurement of Joint Motion: A Guide to Goniometry, 4e. Philadelphia, PA, FA Davis Company. June 2009.

23. Hislop H, Avers D, Brown M. Daniels and Worthingham's Muscle Testing: Techniques of Manual Examination, 9e. W B Saunders Company, 2014.

24. O'Sullivan S, Schmitz T, Fulk G. Physical Rehabilitation: Assessment and Treatment, 6e. Philadelphia, PA. FA Davis Company. 2014. pp. 196-7.

25. Podsiadlo D, Richardson S. The Timed "Up and Go ": A test of basic mobility for frail elderly persons. J Am Geriatr Soc. 1991;39:142-8. [PMID: 1991946] 
26. Binkley JM, Stratford PW, Lott SA, Riddle DL. The Lower Extremity Functional Scale (LEFS): scale development, measurement properties, and clinical application. North American Orthopaedic Rehabilitation Research Network. Phys Ther. 1999;79(4):371-83. [PMID: 10201543]

27. Interactive Guide to Physical Therapist Practice. American Physical Therapy Association. 2003. Published online February 4, 2012. http://guidetoptpractice.apta.org. Accessed February 1, 2013.

28. Light KE, Nuzik S, Personius W, Barstrom A. Low load prolonged stretch vs. high load brief stretch in treating knee contractures. Phys Ther. 1984;64:330-3. [PMID: 6366834]

29. Kisner C, Colby LA. Therapeutic Exercise: Foundations and Techniques, 6e. 2012. pp 190-1.

KEY WORD: Church pew exercises, knee replacement, gait, rehabilitation, physical therapy. 